\title{
Design and Implementation of a High Integrated Noncontact ECG Monitoring Belt *
}

\author{
Fangmin Sun ${ }^{\mathrm{a}, \mathrm{b}, *}$, Zhan Zhao $^{\mathrm{a}}$, Zhen Fang ${ }^{\mathrm{a}}$, Lidong Du ${ }^{\mathrm{a}}$, Diliang Chen ${ }^{\mathrm{a}, \mathrm{b}}$ \\ a State Key Laboratory of Transducer Technology Institute of Electronics, Chinese Academy of \\ Sciences, Beijing 100864, China \\ ${ }^{\mathrm{b}}$ Graduate University of Chinese Academy of Sciences, Beijing 100049, China
}

\begin{abstract}
The ECG plays a key role in the rapid diagnosis of heart diseases such as coronary heart disease, ischemic heart disease, myocardial infarction, arrhythmias, etc. Unfortunately, the adhesion of conventional electrodes to the skin sometimes is difficult if not impossible due to the wet skin caused by higher perspiration. Besides, for the application of long term ECG monitoring, the wet adhesive ECG electrodes are easy to cause infection of the wearers' skin. Moreover, in certain situations it is difficult or simply too time-consuming to undress the patient for an ECG. Therefore, novel ECG monitoring techniques are urgently needed. In this paper, an easy-to-use noncontact ECG (NCECG) monitoring node for wireless body sensor network was designed and tested. The NCECG node introduced in this paper use the doubled shielded active ECG electrodes, compared with many other two electrodes noncontact ECG monitoring node, it also added a right-leg-drive circuit to reduce the common mode noise. The experiment result shows that it could accurately monitor the ECG signals while insulated by one layer of clothes. Furthermore, the NCECG monitoring node we proposed is high compact and easy to use. Besides ECG monitoring function, it also integrates with temperature, respiration and motion state monitoring function, while the size of the circuit board is just $93 \times 40 \mathrm{~mm}$. And the elastic belt architecture makes the ECG monitoring much more convenient and easy to use.
\end{abstract}

Keywords: Active Electrodes; Double Shielded; ECG Monitoring; Wireless Body Sensor Network

\section{Introduction}

With the improvement of the living standards, people are paying more and more attention to their health. The development of the Body Sensor Network (BSN) technology makes it possible to continuous monitor physiological parameters such as Electrocardiograph (ECG) [9], respiratory rate, Electroencephalograph (EEG) [12], arterial oxygen saturation [13], skin temperature, etc, for a long time.

*The paper is based on research funded through 863 Program under Grant No. 2013AA041201.

*Corresponding author.

Email address: sfm0719@163.com (Fangmin Sun). 
The Electrocardiogram (ECG) is a measurement of the electrical activity of the heart over time, captured and externally recorded as measured by various kinds of ECG electrodes. The signals could tell the overall health condition of the heart, the weaknesses in different parts of the heart muscle could be diagnosed in time [1]. This technique is the best way to measure and diagnose abnormal rhythms of the heart, and is commonly used in hospitals all over the world. It is also used in sports and military environments for advanced diagnostics of healthy individuals [14].

Various kinds of electrodes have been used to sample the ECG signal, and the electrodes that are most commonly used to monitor Electrocardiogram (ECG) are gel-type silver/silver chloride $(\mathrm{Ag} / \mathrm{AgCl})$ electrodes. Although such electrodes have been widely used, they are limited in their long-term usage because they usually cause irritation to the skin [3]. Moreover, the gel also dries over time, which will cause a dramatic decrease in the signal quality. Additionally, signal noise is enhanced by the space charge layer at the interface between the skin and the electrode paste. Besides, sweat is another source of signal degradation [4].

Noncontact ECG measurement (NCECG) is a newly developed method that does not require any direct contact between the device and the human skin. And the basic principle of the noncontact measurement of the ECG signal is the capacitive coupling between the skin and a metallic face inside the sensor. These electrodes are placed close to the skin with an insulating material between the electrodes face and the skin. An ultra-high input impedance amplifier is connected to the metallic face to guarantee a low input corner frequency below $100 \mathrm{mHz}$ and a high-input-impedance above 50 G $\Omega$ for the capacitive coupled bio-signal.

Nowadays studies on bio-signal electrodes are many and lots of new types of ECG monitoring electrodes are designed and proposed. Ha-Chul Jung et al. described the fabrication of the PDMS/CNT flexible dry electrodes for long-term ECG monitoring in [5], this kind of electrodes have the advantage of flexible and comfortable for the wearers, nevertheless the electrical property can be affected by many factors such as the density of the CNT, the fabrication techniques, etc. Micro needles designed for bio-signal extracting is described in [2] by Patrick Griss, this kind of electrodes through have comparatively low contact impedance, while, as this kind of electrodes need to insert into the skin, it may cause pain to the wearer whose stratum corneum is thin. Carbon Nanotube (CNT) array designed by Giulio Ruffini in [7] is an improvement method of micro needle. Through the CNT array has wonderful electrical performance and is comfortable for the patient to wear, but the fabrication process of this kind of electrodes is complex. Another kind of dry electrode used in many studies is the active electrode [6], the active electrode is usually consist of a preamplifier circuit and an active shield. This kind of electrode is easy to fabricate and has relatively better signal acquiring performance, so the active electrodes are designed and used in our work.

The main contribution of our work is the design of a noncontact ECG monitoring wearable chest belt which makes it possible to continuous and long-term monitoring of people's health conditions at anytime and anywhere and without causing irritation. Besides, compared with the studies of other noncontact ECG monitoring nodes, the NCECG node we designed has the advantages of small size, low power consumption and easy to use, etc. In order to reduce the common mode interference, a Right Leg Drive (RLD) circuit with the RLD electrode was integrated in the back of the circuit board which could reduce wires to some extent. The picture of the NCECG monitoring belt node is shown in Fig. 1.

The rest of this paper is organized as follows: In Section 2, the design of the double shielded active electrode was introduced. In Section 3, the hardware design of the NCECG belt was de- 


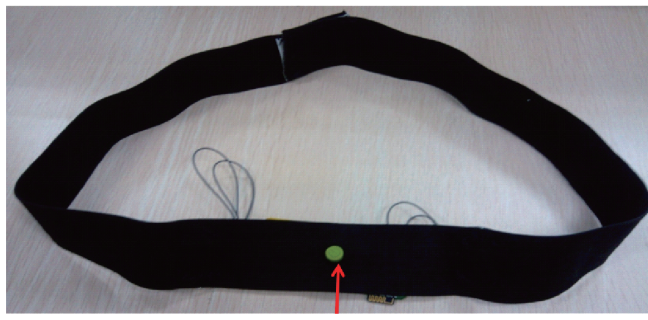

Power switch

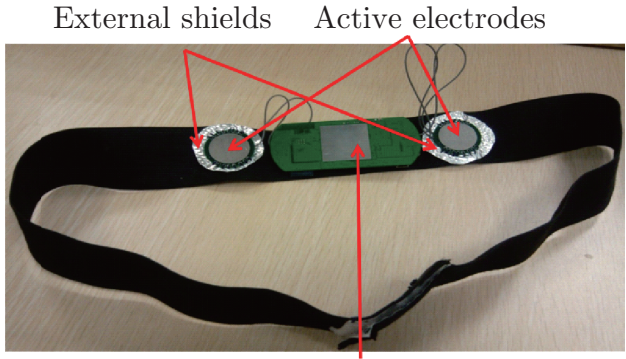

RLD electrode

Fig. 1: The picture of the wearable chest belt. The left is the outside of the ECG monitoring belt, the right is the inside of the ECG monitoring belt

scribed. The performance of the NCECG node is tested and the experiment results are presented in Section 4. The paper was summarized and the future development of the NCECG monitoring belt was described in Section 5 .

\section{Active Electrodes Design}

The design of the electrodes is of much importance for the high accuracy, low noise, and good performance of the ECG monitoring node. According to the measurement principle of the active electrode, the capacitive coupling feature of the electrode is easy to be interfered by the outside environment. Moreover, the contact impedance between the electrode and the skin is usually higher than the contact electrode. So shielding the outside interference and improving the input impedance of the electrode are two key techniques in the design of the active electrode.

The active electrodes are designed to sample the ECG signal indirectly through clothes, and ECG measurement through clothes is characterized by very high electrode impedance (the impedance between the electrode and the skin). To improve the input impedance of the electrode and reduce the noise the following measures were taken in our work: 1) A high impedance front end circuit was designed to improve the input impedance of the electrodes; 2) The double shields were designed and added to the electrodes to reduce the electromagnetism interference from the outside environment. The architecture of the double shielded active electrode is shown in Fig. 2.

In order to make up for the rather high coupling impedance, the amplifying circuits should be as close to the sensing electrodes as possible. For the front end circuit design, each sensor consists of two small round electrically connected standard printed circuit boards and the radius of them is just $15 \mathrm{~mm}$. The upper board contains a high input impedance amplifier LMP7701 which serves as an emitter follower to improve the input impedance, the bottom layer of the board is covered with copper which serves as one of the electrodes of the capacitor made from the skin and the electrode and to sensing the ECG signal.

Also, since capacitive measurements are rather sensitive to noise, these electrodes typically need electromagnetism shielding. A double shielded structure was designed to reduce the external electromagnetism interference: middle layer active shield and external ground shield. The middle layer active shield has the same voltage potential with the sensing layer and could effectively reduce the parasitic capacitance of the sensing layer. The external shield is connected to the ground, which could largely reject the external electromagnetic interference such as the industrial 


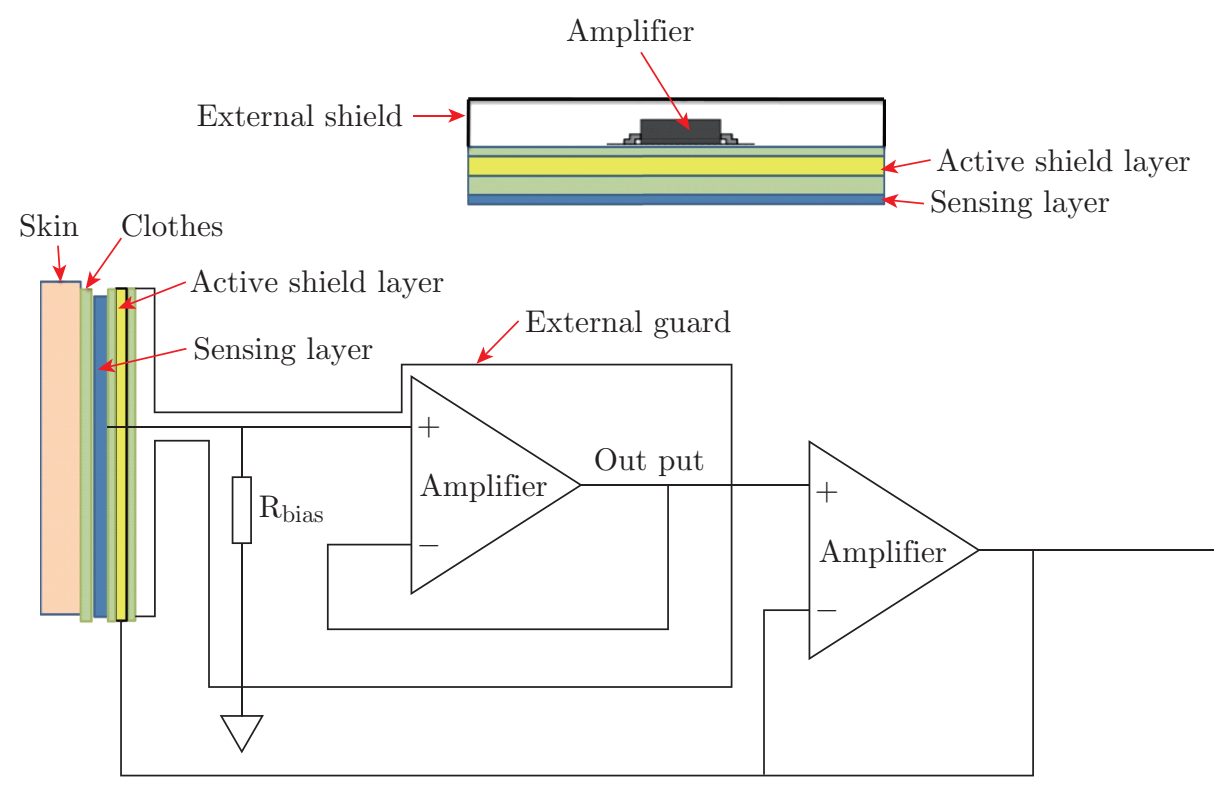

Fig. 2: The model of the double shield active electrode

frequency interference.

\section{Hardware Design of the Node}

It is beneficial for the BSN nodes to be tiny so that they can be easily embedded into a textile or other light-weight wearable devices such as a wrist-worn monitor. In order to have a long lifetime, each component of the system should consume as little power as possible. Small size and low power consumption should always be put in mind in the design of the node. The hardware framework of the NCECG monitoring node is depicted in Fig. 3. It mainly includes four function modules: microcontroller module, sensing and signal processing module, wireless communication module and power management module. In this section, these modules will be discussed respectively.

\subsection{Sensing Module}

As introduced previously, the NCECG monitoring node not only could accurately sample the ECG signal but also could monitoring the body temperature and the motion state of the wearer. The ECG signal is sensed by the double shielded active electrodes closed to the skin and a right leg driven electrode is added to reduce the common interference.

Body temperature is also an important factor for the diagnosis of many diseases, the NCECG monitoring node also integrated a body temperature sensor for continuous monitoring of the skin temperature. For the temperature measurement, the YSI4000 family temperature sensor is selected to capture the skin temperature signal, and the AD7783 is chosen for the temperature measurement, which adopts the ratio metric method. The transducer excitation (voltage or current) on the analog input drives the reference voltage for this part, thus the effect of the low frequency noise in the excitation source can be removed.

In order to evaluate, select and self-correct the monitoring parameters according to the motion 


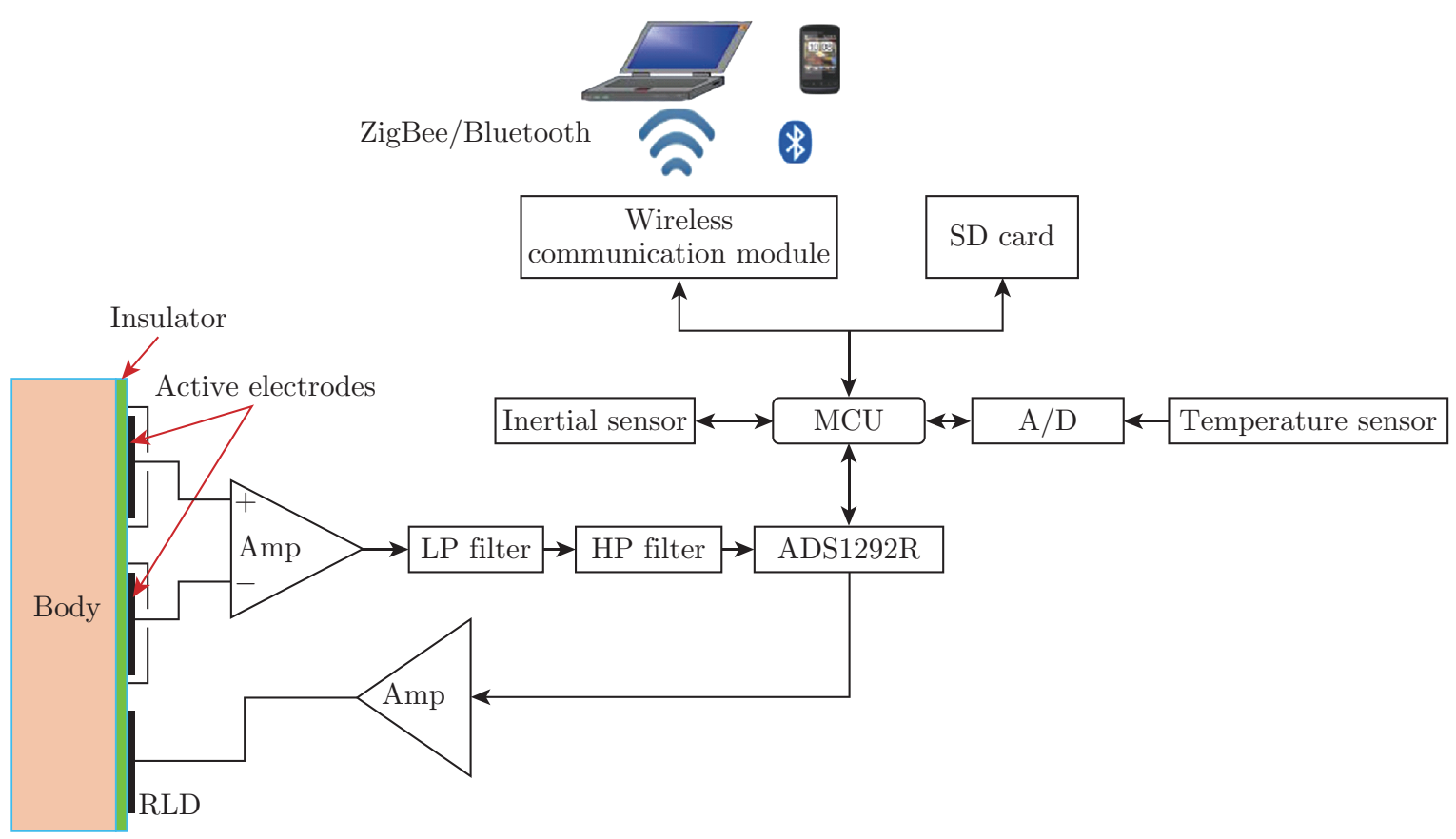

Fig. 3: The architecture of the NCECG monitoring node

state of the wearer, which can avoid and reduce the interference caused by daily activity on the physiological signal measuring, and can improve the monitoring quality and health expert diagnostic efficiency, we integrated an inertial sensor CMA3000D01 in the ECG monitoring node for real-time monitoring of the wearer's behavioral state.

\subsection{Signal Processing Module}

The ECG signal sampled from the active electrodes is first be filtered by a $100 \mathrm{~Hz}$ LP filter and a $0.05 \mathrm{~Hz} \mathrm{HP}$ filter, besides, a notch filter whose notch frequency is $50 \mathrm{~Hz}$ is designed to reduce the power line interference. And then the filtered signal is converted into digital signal by the Analog/digital device.

The Analog to Digital Converter (ADC) was chosed for the node mainly based on the following principles: 1) Ultra low power consumption; 2) High resolution; 3) Highly integrated; 4) Small size. According to these principles, we finally adopt TI's 24 bit ADS1292R as the ADC. The ADS1292R incorporate all of the features that are required in our design of a compact and lowpower ECG and respiration monitoring node. With its exceptional performance and high levels of integration, the ADS1292R make the front-end ECG signal processing circuits very simple and largely reduced the physical size and power consumption of the nodes. As previously mentioned, the ADC digital data lines are connected as a daisy chain shifting serial data from the end of the sensor chain back to the host data acquisition module. A common clock and chip select line synchronizes the conversion and transfer of data at a sample rate of 500 sps.

However, the ECG signal that the microcontroller received is not ideal because of the serious baseline drift. Baseline drift, if not removed, could influence the accurate diagnosis of heart disease such as ischemia and arrhythmia. In the post-software processing module, a simple, practical and fast shifting window median filtering algorithm is used to reduce the baseline drift without degrading original ECG signal [8]. 


\subsection{Wireless Communication Module}

The sensor was integrated with two types of wireless communication modules: the Zigbee and the Bluetooth. The Zigbee transceiver circuit is made up of the radio of the CC2530 chip and a printed circuit antenna. The radio of the CC2530 chip can easily be built on top of the IEEE 802.15.4 based standard protocols such as RemoTI, TIMAC, and Z-Stack. However, take the power consumption of these protocols into consideration, we finally choose the Z-Stack protocol as our application platform [11]. For the design of the antenna, a small $2.4 \mathrm{GHz}$ antenna was made in the printed circuit board which largely reduced the size of the node compared with the whip antenna. To realize the communication between the node and other Bluetooth devices such as mobile phones, the node is also integrated a Bluetooth module. With these two communication methods, the physiological information can be easily send to the remote data base for storage or clinical center for pathology analysis through the Zigbee, or just send to a nearby Bluetooth device to be shown [10].

\subsection{Power Management Module}

To improve the energy efficiency, a Dynamic Power Management (DPM) module and a Dynamic Voltage Scaling (DVS) module were added to the ECG monitoring node operation system, which would make the node using its resources more efficiently. When there is nothing interesting happen around the node, some modules of the nodes are in the idle mode, and the DPM module will turn off these modules or turn them into sleep mode to save more energy. The DVS module calculates the loads of the power source, and when the loads is lower than the threshold it will reduce the work frequency and voltage of the microprocessor to reduce the process ability of the microprocessor module and thus reduce the power consumption of the processor.

In the low power mode which is set by the button in the device, the wireless transmission module is closed and the physiological signals could be stored in the mini SD card automatically, relative people could read the SD card when needed. This largely reduced the power consumption of the sensor node. And when the NCECG node is not in use, it could be set into the sleep mode through push the button longer, and in the sleep mode the MCU is in the low-power mode and the sensing module and the wireless communication module are all suspended. In the same way, the device can be waked up by long time pushing the button.

\section{Experiment Results and Conclusions}

In this section, the performance of the NCECG node included the frequency response of the electrode, the power consumption of the node and the monitoring function of the node is tested, and the results are given and analyzed.

\subsection{The Frequency Response of the Electrode}

To know the frequency response of the active electrode, a differential test frequency sweep was applied to the metal plates from a function generator. The sensor output, along with the test 
input was recorded through a digital oscillograph, allowing the gain to be measured. In this experiment, the insulator medium between the electrode metal plate and the function generator electrode is a piece of cotton cloth with the thick of $1.2 \mu \mathrm{m}$, and the test result is shown in Fig. 4 . As the frequency of the ECG signal is mainly ranging from $2 \mathrm{~Hz}$ to $20 \mathrm{~Hz}$, so we can see through the result that active electrode has a very good performance to sample the human ECG signal.

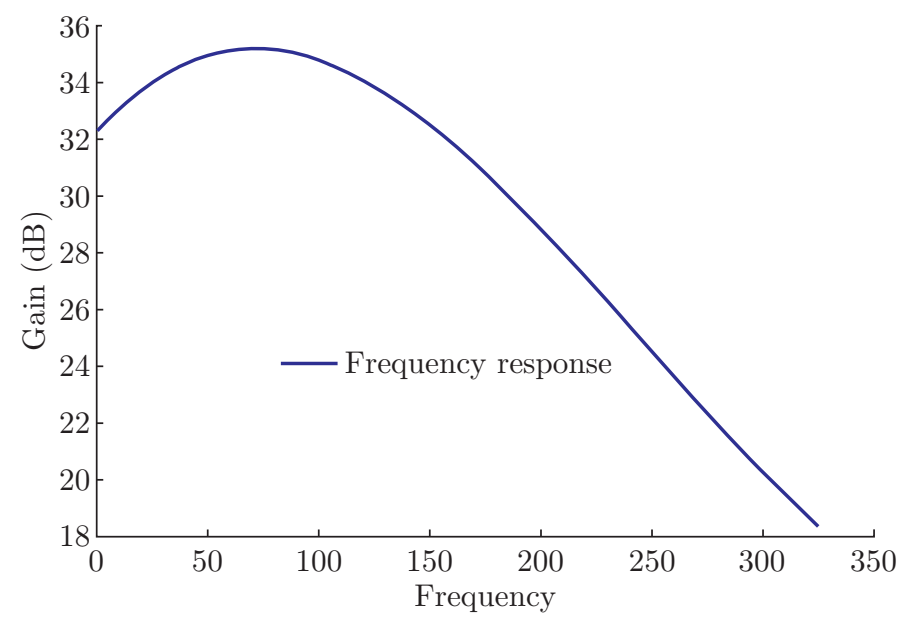

Fig. 4: The frequency response of the active electrode (insulator medium: cotton)

\subsection{The Health Monitoring Function of the NCECG Node}

Different experiments were done to verify the ECG monitoring performance of the designed NCECG. 1. Direct contact mode. The ECG is tested when the active electrodes in direct contact with the skin. One of my colleagues wears the NCECG belt with his chest naked. And the direct contact testing result is shown in Fig. 5, and the P-wave, QRS-wave and the T-wave can be clearly seen in the result. 2. The non-contact testing mode. The ECG signal was sampled from the chest over a cotton $\mathrm{T}$ shirt which is about $10 \mathrm{um}$ thick. The noncontact test result is shown in Fig. 6, and the QRS-wave and the T-wave can be detected in this condition. 3. The noncontact and in motion testing mode. The ECG signal was sampled from the chest over a cotton $\mathrm{T}$ shirt which is about $10 \mathrm{um}$ thick while the people being monitored was quickly walking. The test result is shown in Fig. 7, the noncontact ECG signal tested when the people is in the motion state mixed with strong high-frequency noise signal. A FIR filter was designed to filter out the noise and the filtered signal is shown in Fig. 8.

The LabView based interface is designed in the personal computer to further process the sam-

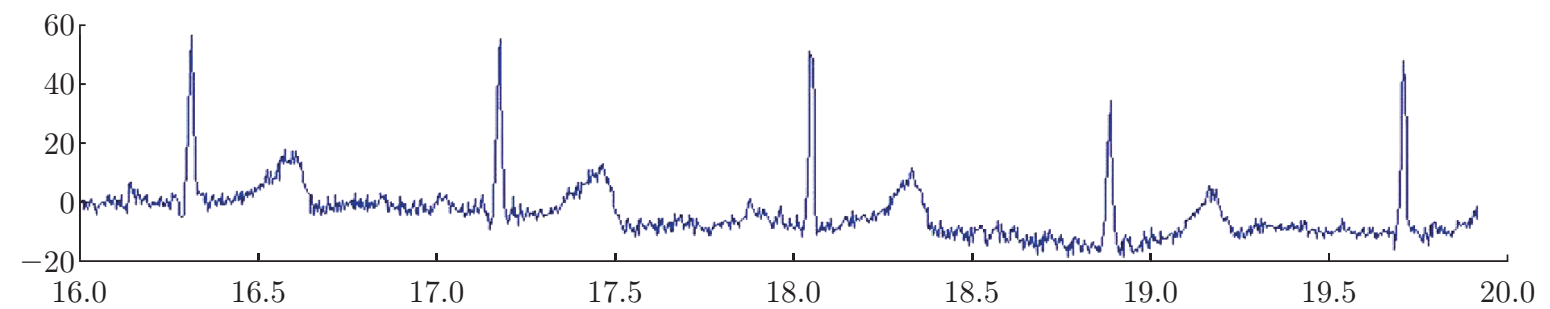

Fig. 5: The tested ECG in the case that the active electrodes in directly contact with the skin 


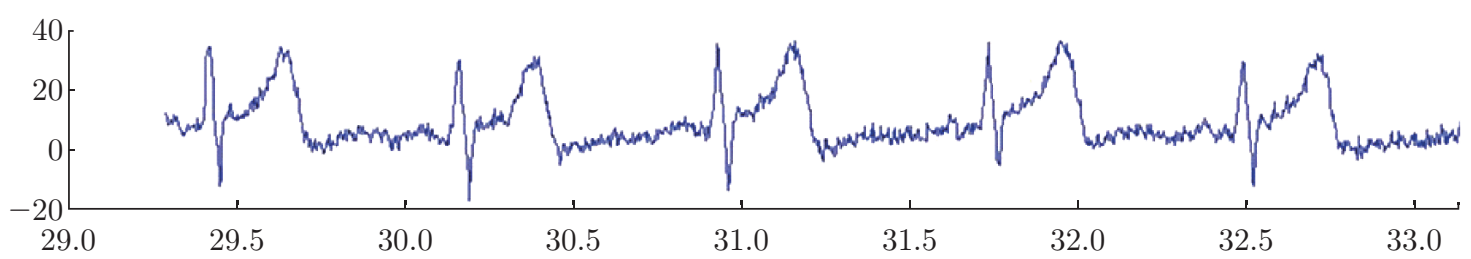

Fig. 6: The tested ECG in the case that the active electrodes sample the ECG signal through a T shirt which is about 10 um thick

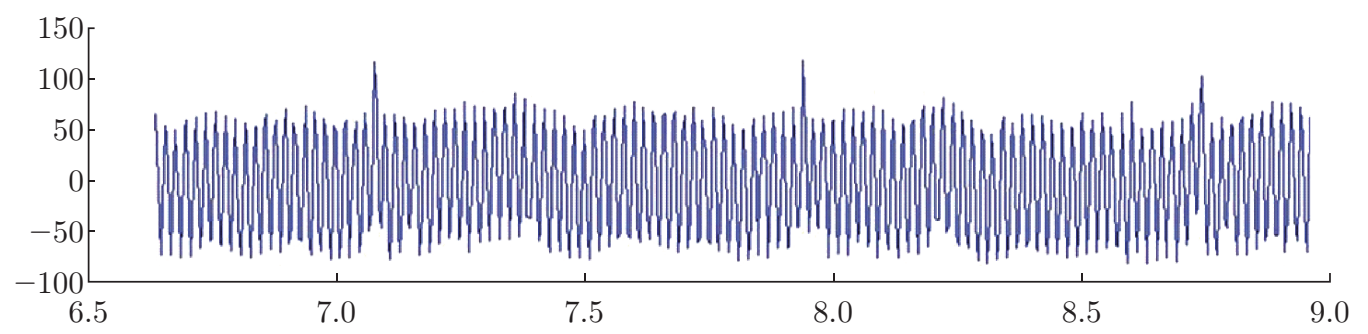

Fig. 7: The original NCECG signal sampled from the chest over a cotton $\mathrm{T}$ shirt while the people monitored was quickly walking

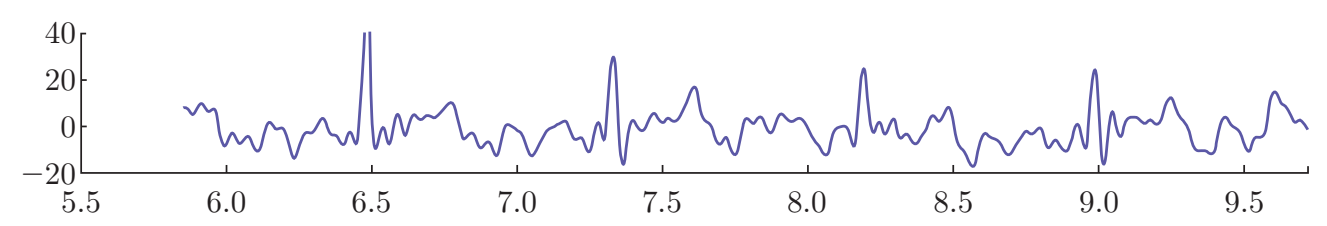

Fig. 8: The filtered signal of the NCECG signal shown in Fig. 7

pled ECG signal and show the test results of the ECG, temperature and the acceleration (see Fig. 9).

A clear ECG signal could be observed when the sensor is placed directly above the skin's surface. However, when the recording is made over insulated medium, some $50 \mathrm{~Hz}$ noise is easy to be introduced as a result of capacitive mismatch due to the larger separation distance and
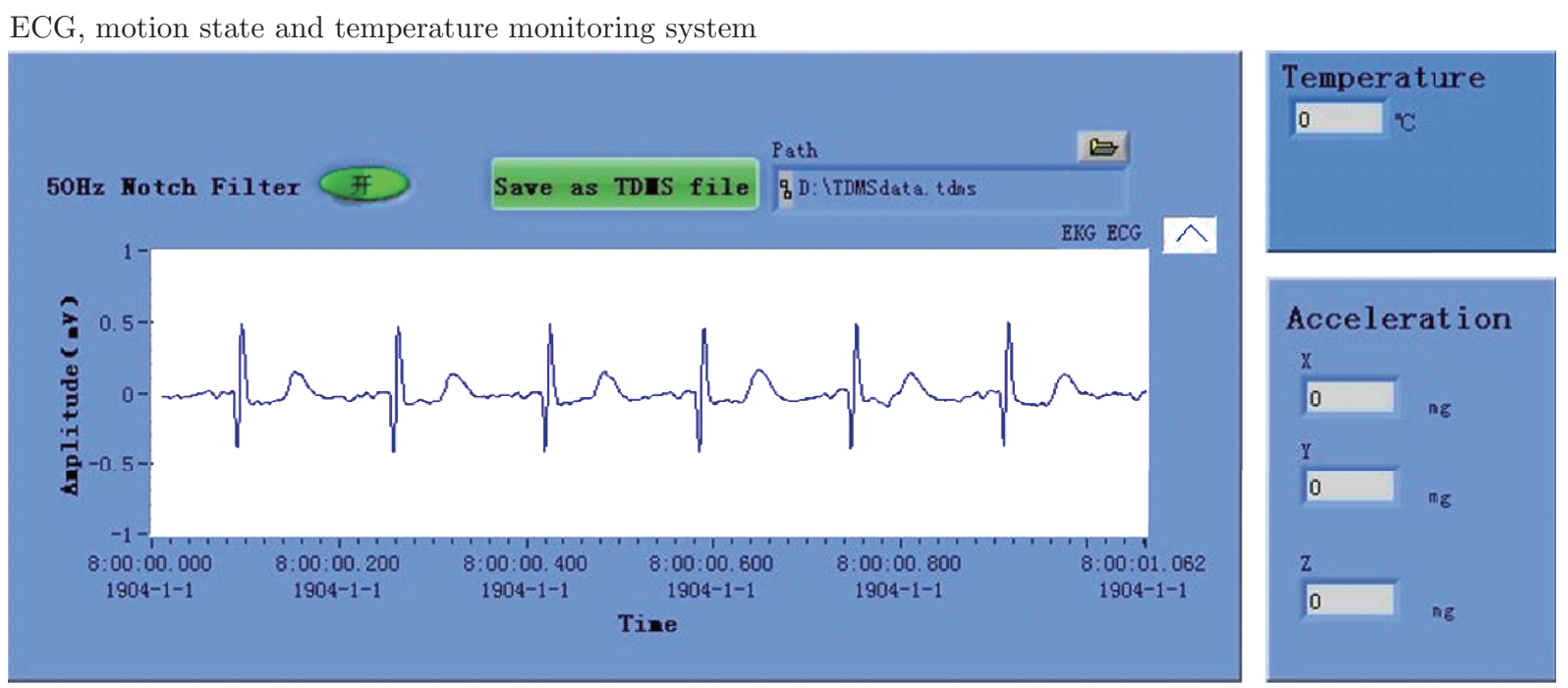

Fig. 9: The LabView based interface in personal computer 
corresponding smaller coupling capacitance. To reduce the $50 \mathrm{~Hz}$ power line interference, a 50 $\mathrm{Hz}$ notch filter was designed with the labView software.

\section{Conclusion}

This paper described the design of a compact, low-power, small-size and high-integrated health monitoring sensor node which could be used to monitor multi physiological signals of human comfortably for a long time. We mainly discussed the double shielded active electrode design and the hardware design of the electric circuit. At the end, the frequency response of the active electrode was studied and the functions of the node have been tested. Experiments to analyze the power consumption of the node when it is in different mode was done. The test result shows that this high compact noncontact ECG monitoring node could accurately measure the wearer's ECG signal, body temperature and motion state while consume relatively lower power.

\section{References}

[1] Adrian Sapio, Gill R. Tsouri, Low-power body sensor network for wireless ECG based on relaying of creeping waves at $2.4 \mathrm{GHz}, 2010$ International Conference on Body Sensor Networks, 167-173

[2] Patrick Griss, Peter Enoksson, Heli K. Tolvanen-Laakso, Pekka Meriläinen, Stig Ollmar, Göran Stemme, Micromachined electrodes for biopotential measurements, Journal of Microelectromechanical Systems, Vol. 10, No. 1, March 2001, 10-16

[3] A. Searle, L. Kirkup, A direct comparison of wet, dry and insulating bioelectric recording electrodes, Physiol. Meas., Vol. 21, 2000, 271-283

[4] B. A. Taheri, R. T. Knight, R. L. Smith, A dry electrode for EEG recording, Electroencephalogr. Clin. Neurophysiol., Vol. 90, No. 5, 1994, 376-383

[5] Jae Hee Lee, Young Woo Nam, Ha-chul Jung, Dong-hyun Baek, Sang-hoon Lee, Joung Sook Hong, Shear induced CNT/PDMS conducting thin film for electrode cardiogram (ECG) electrode, BioChip J., 2012, 91-98

[6] Yu M. Chi, Stephen R. Deiss, Gert Cauwenberghs, Non-contact low power EEG/ECG electrode for high density wearable biopotential sensor networks, 2009 Body Sensor Networks, 246-250

[7] Giulio Ruffini, Stephen Dunne, Esteve Farr'es, Josep Marco-Pallar'es, Chris Ray, Ernest Mendoza, Ravi Silva, Carles Grau, A dry electrophysiology electrode using CNT arrays, Sensors and Actuators A, 132, 2006, 34-41

[8] Fangmin Sun, Zhan Zhao, Zhen Fang, Yaohong Shi, Xianxiang Chen, Yundong Xuan, A design of a Band-Aid like health monitoring node for body sensor network, 2012 International Conference on Measurement, Information and Control (MIC), 2012, 34-39

[9] Shubhada Ardhapurkar, Ramchandra Manthalkar and Suhas Gajre, ECG denoising by modeling wavelet sub-band coefficients using kernel density estimation, Journal of Information Processing Systems, Vol. 8, No. 4, 2012, 669-684

[10] Koohong Kang, Performance anomaly of the IEEE 802.11 DCF in different frame error rate conditions, Journal of Information Processing Systems, Vol. 8, No. 4, 2012, 739-0

[11] Reen-cheng Wang, Ruay-shiung Chang, Jei-hsiang Yen, Pu-I Lee, A dynamic topology reformation algorithm for power saving in ZigBee sensor networks, International Journal of Distributed Sensor Networks, Vol. 2013, 2013, 1-11 
[12] Yunsick Sung, Kyungeun Cho, Kyhyun Um, A framework for processing brain waves used in a brain-computer interface, Journal of Information Processing Systems, Vol. 8, No. 2, 2012, 315-330

[13] Junho Ahn, Richard Han, An indoor augmented-reality evacuation system for the smartphone using personalized pedometry, Human-centric Computing and Information Sciences, 2012

[14] Xiong Yang, Gang Peng, Zhaoquan Cai, Kehan Zeng, Occluded and low resolution face detection with hierarchical deformable model, Journal of Convergence, Vol. 4, No. 2, June 2013

[15] Sean Thorpe, Virtual machine history model framework for a data cloud digital investigation, Journal of Convergence, Vol. 3, No. 4, December 2012 